\title{
EFFECTS OF DAMAGE TO THE SUPRACHIASMATIC AREA OF THE ANTERIOR HYPOTHALAMUS ON THE DAILY MELATONIN AND CORTISOL RHYTHMS IN THE RHESUS MONKEY ${ }^{1}$
}

\author{
STEVEN M. REPPERT,,$^{*, 2}$ MARK J. PERLOW,$\neq^{3}$ LESLIE G. UNGERLEIDER, $\S$ \\ MORTIMER MISHKIN,§ LAWRENCE TAMARKIN,* DAVID G. ORLOFF, ${ }^{*}$ HOWARD J. HOFFMAN, \\ AND DAVID C. KLEIN*
}

"Section on Neuroendocrinology, Laboralory of Developmental Neurobiology, National Institule of Child Heallh und Human Development, National Institutes of Health, Bethesda, Maryland 20205; $\ddagger$ Laboratory of Clinical Psychopharmacology, National Institute of Mental Health, Washington, DC 20052; §Laboratory of Neuropsychology, National Institute of Mental Health, Bethesda, Maryland 20205; and $\rceil$ Epidemiology and Biometry Research Program, National Institute of Child Health and Human Development, National Institutes of Health,

Bethesda, Maryland 20205

\begin{abstract}
The effects of lesions of the suprachiasmatic nucleus (SCN) on the circadian rhythms in melatonin and cortisol were examined in the rhesus monkey. The concentrations of the two hormones were monitored in cerebrospinal fluid (CSF) withdrawn from two sham-operated animals, two animals with complete bilateral SCN lesions, and two animals with partial SCN damage at 4 and 8 months after surgery. In the sham-operated animals, as in the intact animal, the daily melatonin rhythm was entrained to the daily light-dark cycle, was suppressed in constant light, and persisted in constant darkness. In contrast, neither animal with complete SCN ablation exhibited a daily pattern of CSF melatonin in diurnal lighting at 4 months after surgery nor were their melatonin levels at constant low values. Furthermore, CSF melatonin concentrations were not suppressed in either animal by constant light. Surprisingly, at 8 months after surgery, spectral analysis revealed a $24-\mathrm{hr}$ component to the melatonin patterns for each animal with complete SCN ablation in both diurnal lighting and constant darkness. The two animals with partial SCN damage exhibited a daily melatonin rhythm in diurnal lighting, but constant light did not suppress CSF melatonin concentrations consistently. Daily rhythms persisted in both for a $6 \frac{1}{2} 2-\mathrm{d}$ period of study in constant darkness. In contrast to the alterations in the melatonin rhythm after SCN damage, there was no apparent effect of either partial or complete SCN ablation on the daily CSF cortisol rhythm. These data indicate that, in the rhesus monkey, the SCN is important for the generation, photic entrainment, and photic suppression of the melatonin rhythm. However, circadian oscillators located outside of the SCN region may control the normal daily cortisol rhythm and perhaps the melatonin rhythm in the absence of the SCN.
\end{abstract}

A growing body of evidence indicates that in rodents the suprachiasmatic nucleus (SCN) of the anterior hypothalamus plays a key role in the circadian time-keeping system and functions as a biological clock or endogenous oscillator (Rusak and Zucker, 1979). This clock is reset on a daily basis by environmental lighting, which acts via

\footnotetext{
${ }^{1}$ We thank Thelma W. Galkin for preparation of the photomicrographs, Dr. Yoshitaka Ohnishi of the National Eye Institute (current address: Kyushu University, Fukuoka, Japan) for assistance with the intraocular injections, and Kathleen Sullivan for clerical aid.

${ }^{2}$ To whom correspondence should be addressed at his present address: Children's Service, Massachusetts General Hospital, Boston, MA 02114

${ }^{3}$ Present address: Department of Neurology, Mt. Sinai Medical Center, New York, NY 10029.
}

the eye and a retinohypothalamic projection (RHP) to the SCN (Moore, 1979). This mechanism insures that a number of 24-hr rhythms in physiological function are coordinated with the environmental lighting schedule.

We have been interested in circadian rhythms in the pineal hormone, melatonin, and the adrenocorticoid, cortisol, in the rhesus monkey (Reppert et al., 1979, 1980; Perlow et al., 1980, 1981). Since little is known about the relationship of these rhythms to the SCN-RHP complex in the primate, we examined the effects of ablation of the SCN-RHP complex on the two hormone rhythms in the rhesus monkey.

\section{Materials and Methods}

Animals. Male rhesus monkeys (Macaca mulatta) were obtained from the National Institutes of Health 
primate colony. Two adult animals $(5.8$ and $6.2 \mathrm{~kg}$, respectively) were used for preliminary histological studies. Six adolescent animals (range, 3.5 to $4.5 \mathrm{~kg}$ ) were used for the physiological investigations. When the animals were not in restraining chairs (see below), they were maintained in individual cages in a room with automatically regulated lighting (light:dark (LD) 12:12), with lights on at $0600 \mathrm{hr}$. Food (Purina Monkey Chow) and water were available ad libitum.

Surgical procedures. Four of the adolescent animals $(564,583,585$, and 586$)$ received bilateral lesions of the hypothalamus in the area of the SCN. The two others (565 and 580) received sham operations, which were identical to the experimental procedures required for the lesions except that brain tissue was not removed.

Anesthesia was accomplished with ketamine hydrochloride $(10 \mathrm{mg} / \mathrm{kg})$ and sodium pentobarbital $(30 \mathrm{mg} /$ $\mathrm{kg})$. The animals also received atropine sulfate $(0.1 \mathrm{mg})$ and dexamethasone phosphate $(2.0 \mathrm{mg})$. During the surgical procedures, heads were secured in a head holder.

An area of frontal bone over the left orbit was removed, a dural flap was turned, and the left orbital frontal cortex was raised slightly with a brain retractor. The triangular area of tissue between the optic chiasm and the confluence of the left and right anterior communicating arteries was identified through a surgical microscope, and gray matter at the base of this triangle immediately above the chiasm and on either side of the midline then was removed by aspiration with a small gauge metal suction tube. The lesion was intended to extend approximately $1.5 \mathrm{~mm}$ dorsally from the top of the chiasm, $1.5 \mathrm{~mm}$ laterally from either side of the midline, and $2 \mathrm{~mm}$ posteriorly from the front of the chiasm. Following surgical removal, the dura, subcutaneous tissues, and scalp were closed with silk sutures, the wound was sprayed with furazolidone, and the animal was given long acting Bicillin (600,000 units).

Experimental observations. Experimental observations were made at two times, 4 and 8 months after surgery. In both cases, the same adaptation and sampling procedures were followed. Animals were adapted to chronic restraint in primate chairs during a 2- to 3-week period. Each chair was housed in an isolation chamber which insured a well ventilated, sound-attenuated environment; lighting was automatically regulated (Perlow et al., 1979; Reppert et al., 1979). Unless otherwise indicated, the lighting schedule was LD 12:12, with the light period starting at $0600 \mathrm{hr}$. Light was provided by a GE Cool White fluorescent tube; intensity at the level of the animal's head was 450 lux.

On each day between 1000 and $1200 \mathrm{hr}$, food was placed on the feeding trays and routine care was performed. Water was available ad libitum. During constant dark experiments, daily care was provided using a low intensity red light.

After adaptation, each animal was anesthetized and fitted with an indwelling subarachnoid catheter, terminating in the cisternal region. Cerebrospinal fluid (CSF) was removed automatically $(1 \mathrm{ml} / \mathrm{hr})$ in $2-\mathrm{hr}$ fractions and stored at $-20^{\circ} \mathrm{C}$ until analysis. This procedure has been described previously (Reppert et al., 1979).

Histological analysis. Seven days prior to killing, each of the adolescent animals with lesions was given a $50-\mu \mathrm{l}$ intraocular injection of saline containing $250 \mu \mathrm{Ci}$ of $\mathrm{L}$ $\left[2,3,4,5-{ }^{3} \mathrm{H}\right]$ proline $(115.4 \mathrm{Ci} / \mathrm{mmol}$, New England $\mathrm{Nu}-$ clear) and $250 \mu \mathrm{Ci}$ of $\mathrm{L}-\left[3,4,5-{ }^{3} \mathrm{H}(\mathrm{N})\right]$ leucine $(111.2 \mathrm{Ci} /$ mmol, New England Nuclear) at a concentration of 10 $\mu \mathrm{Ci} / \mu \mathrm{l}$. Injections were made through a 26 gauge needle using a Hamilton microliter syringe. The material was placed in the vitreous humor of both eyes of the four experimental adolescent animals and one adult (267) and in one eye of the other adult monkey (43).

Seven days later, the animals were given a lethal dose of anesthetic and perfused transcardially with $0.9 \%$ saline followed by $10 \%$ formol/saline. The brains were removed, photographed, blocked stereotaxically in the frontal plane, and placed in a solution of $30 \%$ sucrose in $10 \%$ formol/saline until they sank. Frozen bilateral sections then were cut coronally at $30 \mu \mathrm{m}$, and every fifth section was processed for autoradiography (Cowan et al., 1972). Sections were exposed to Kodak NTB-2 nuclear track emulsion in light-tight boxes for $28 \mathrm{~d}$ at $4^{\circ} \mathrm{C}$. They were developed in Kodak D-19 at $19^{\circ} \mathrm{C}$ and counterstained with thionin prior to microscopic study of radioactively labeled nerve fibers.

For the experimental animals, lesions were evaluated under bright-field illumination to determine their precise location and extent; sparing of the retinal projection to the SCN was determined under dark-field illumination. For the normal animals, the distribution and density of silver grains within the SCN were charted onto enlarged drawings, and this information was correlated with architectural subdivisions of the nucleus. No histological data were obtained from the two sham-operated control animals.

Melatonin radioimmunoassay (RIA). The concentration of melatonin in each CSF fraction was determined using a modification (Reppert et al., 1979) of the Rollag and Niswender (1976) RIA technique. The CSF samples of each animal for each of the two sampling periods were analyzed in the same assay run. The within and between assay coefficients of variation were $10 \%$ and $20 \%$, respectively. The limit of assay sensitivity was $1.0 \mathrm{pg} / \mathrm{ml}$.

Cortisol RIA. Cortisol concentration in CSF was measured in unextracted samples by Hazelton Laboratories (Vienna, VA). CSF samples of each animal for each of the two sampling periods were analyzed in the same assay run. The within and between assay coefficients of variation were $10 \%$. The limit of assay sensitivity was 0.1 $\mu \mathrm{g} / \mathrm{dl}$.

Experimental protocols. At 4 months after the operation, CSF was collected from each animal for several days in diurnal lighting, $2^{1 / 2} \mathrm{~d}$ in constant light and $2^{1 / 2} \mathrm{~d}$ in constant darkness. At 8 months postoperatively, CSF was collected continuously from each animal for several days in diurnal lighting and for $6^{1 / 2} \mathrm{~d}$ in constant darkness.

Statistical methods. Relative amplitude and phase angle diagrams were produced using the technique of complex demodulation (Orr and Hoffman, 1974; Bloomfield, 1976). In this approach, standard low pass filters of the moving average type are used to smooth the amplitude and phase information for the frequency band of interest $(1 \mathrm{cycle} / \mathrm{d})$. Prior to the smoothing operation, the frequency band centered at $1 \mathrm{cycle} / \mathrm{d}$ is exchanged with the frequency band centered on the lowest frequency, 0 , which corresponds to an arbitrarily large pe- 
riod in cycles per day. The corresponding bandpass filter output can be easily produced by remodulation, but because of limitations on space, these figures were not included.

The power spectra were computed with a fast Fourier transform algorithm (Gentleman and Sande, 1966), and then a modified periodogram estimate of the spectrum was formed (Bingham et al., 1967; Orr and Hoffman, 1974). This method of estimating power spectra provides high resolution and is relatively bias free.

\section{Results}

\section{Histology of the SCN and RHP in the normal monkey}

The SCN is easily identified histologically just above the optic chiasm, ventrolateral to the base of the third ventricle in the anterior hypothalamus. The nucleus is ovoid in shape and consists of small, densely packed cells. The cell density is highest in the ventrolateral portion of the nucleus where the cells are darkly staining (see Fig. $1 B)$. Medial and dorsal to the darkly staining cells, there is a gradual decline in cell density, resulting in a somewhat indistinct boundary along these portions of the nucleus.
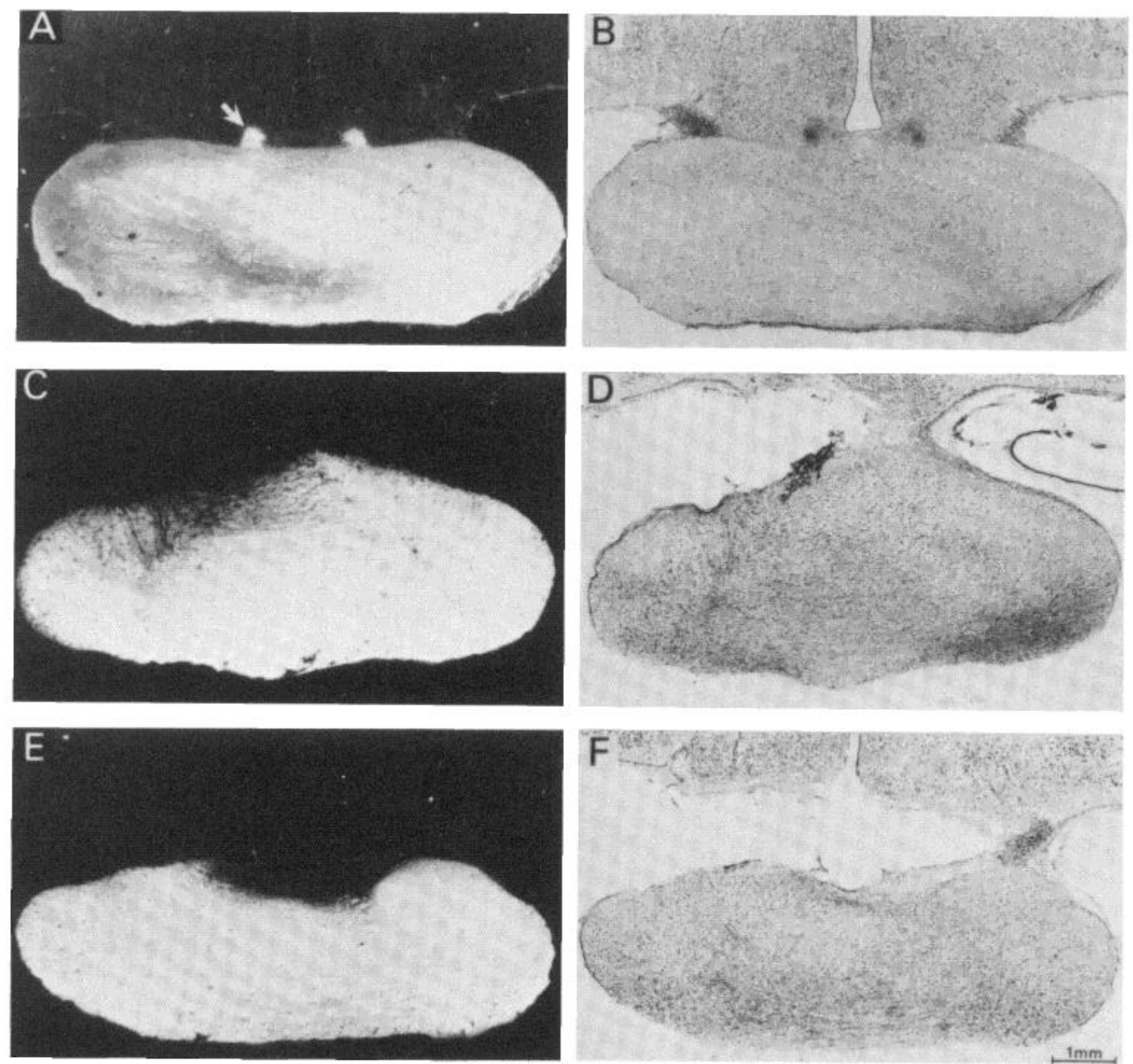

Figure 1. Dark-field $(A)$ and bright-field $(B)$ photomicrographs of a coronal section through the suprachiasmatic nucleus (SCN) of case 267, a normal rhesus monkey given a binocular injection of tritiated amino acids. The arrow in $A$ indicates radioactive label within the left SCN. $C$ to $F$, Dark-field and bright-field photomicrographs of coronal sections through the greatest extent of damage in two experimental monkeys (case 583, $C$ and $D$; case $586, E$ and $F$ ). The absence of radioactive label dorsal to the chiasm confirms the cell stain evidence of bilateral destruction of the SCN in both cases. The scale in $F$ applies to $A$ to $F$.
Autoradiographic results from animals 267 and 43, the two normal monkeys given intraocular injections, confirmed the direct retinal projection to the SCN. In both cases, radioactively labeled fibers coursing through the chiasm entered the SCN from its ventrolateral aspect and terminated preferentially and densely in the adjacent portion of the nucleus, the portion containing the darkly staining cells (see Fig. $1 A$ ). Outside of this subdivision, the nucleus received only a patchy input. Based on areal measurements, about $50 \%$ of the SCN receives a direct retinal projection. In contrast to other species, in which the retinal projection to the SCN is heaviest in the caudal one-third to one-half of the nucleus (Moore, 1973), we found label distributed uniformly from the anterior to the posterior limit of the SCN. The results from animal 43 , the monocularly injected case, confirmed prior findings that the RHP in the monkey is bilateral, though asymmetrical, with the contralateral SCN receiving the heavier input (Moore, 1973).

\section{Histology of the experimental brains}

Histological examination of the four experimental brains under bright-field illumination revealed that, as 
intended, the lesions were uniformly small and restricted mainly to the area of the SCN. In all cases, the lesions extended from the rostral portion of the chiasm posteriorly for about $2.5 \mathrm{~mm}$ to the border of the retrochiasmatic area.

Animals with complete SCN ablation. In animals 583 and 586 , the lesions completely destroyed the SCN bilaterally, encroaching only minimally on the dorsal limit of the chiasm; coronal sections through the greatest extent of damage for these two animals are shown in Fig. 1, $C$ to $F$. In both, the damage outside of the SCN was asymmetrical. Thus, whereas, on the right side of animal 583, the damage was confined almost completely to the SCN, on the left side, there was additional damage to the ventral part of the preoptic area and almost complete destruction of the supraoptic nucleus (Fig. $1 D$ ). In animal 586 , similarly, the lesion outside of the SCN on the right side was minimal, involving damage only to the anterior part of the supraoptic nucleus, whereas, on the left side, the supraoptic nucleus was removed completely and the ventral part of the preoptic area was invaded (Fig. $1 F$ ). Autoradiographic results from these two animals (583, Fig. $1 C$; 586, Fig. $1 E$ ) revealed no trace of label in the hypothalamic area dorsal to the densely labeled chiasm, confirming the cell stain evidence of complete bilateral destruction of the RHP to the SCN in both animals.

Animals with partial SCN ablation. The two other experimental animals, 564 and 585, had partial unilateral sparing of the SCN. In animal 564, the SCN was destroyed completely on the right side but only incompletely on the left (see Fig. 2). This animal also sustained bilateral, though minimal, damage to the ventral part of the preoptic area; on the left side, this damage extended posteriorly to include the rostral tip of the ventromedial hypothalamus. The optic chiasm and the supraoptic nuclei were totally spared. Autoradiographic results confirmed the cell stain evidence of complete removal of the RHP to the SCN on the right; on the left side, however, the location of label above the chiasm indicated that the anterior part of the projection to the SCN had been spared. The spared portion was estimated to comprise approximately $35 \%$ of the projection zone within the left SCN.

In animal 585 , the lesion was bilaterally symmetrical and, under bright-field illumination, the SCN erroneously appeared to be totally destroyed (see Fig. $3 B$ ). The lesion encroached on the dorsal edge of the chiasm at the midline and the most ventral part of the preoptic area
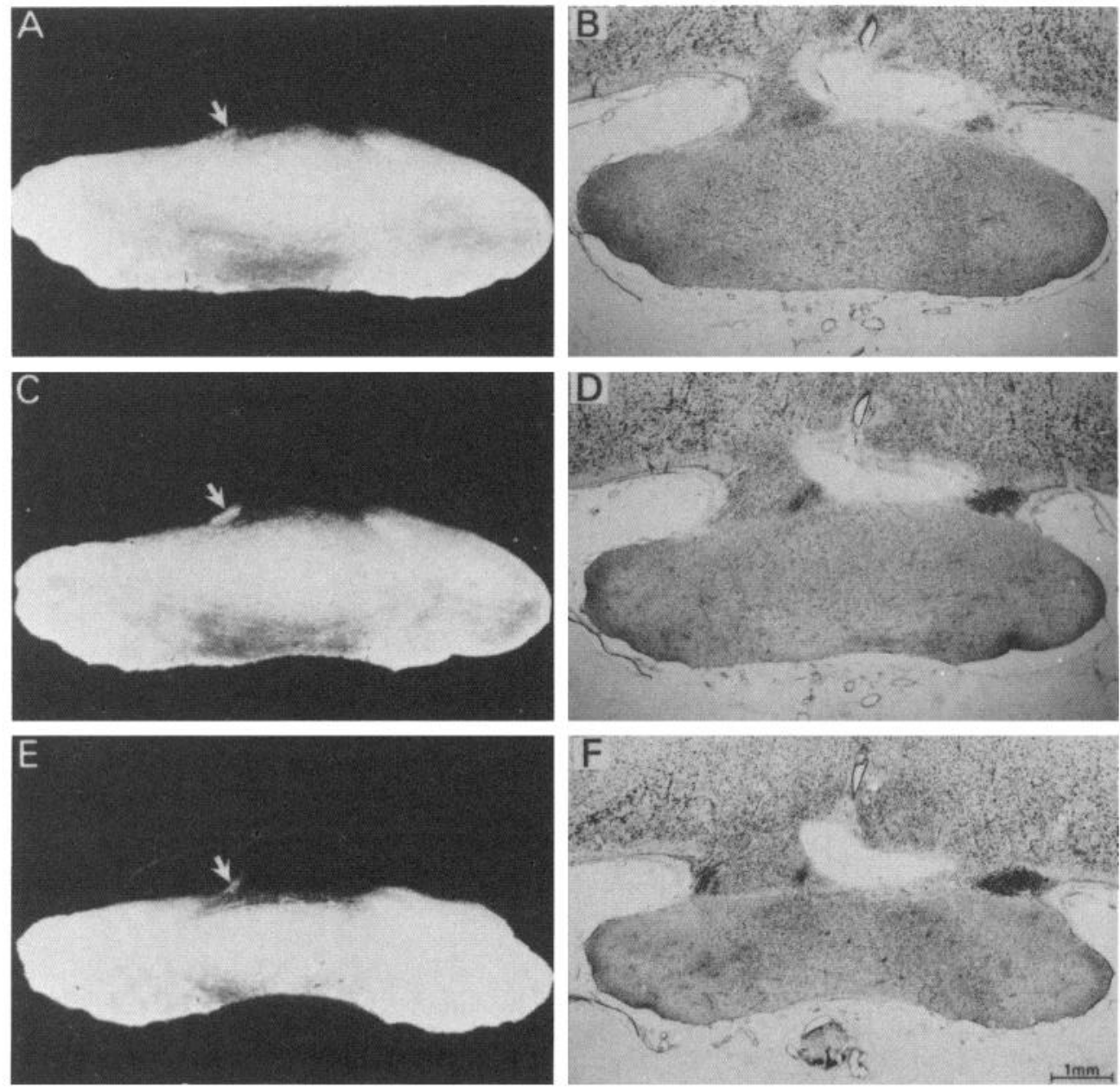

Figure 2. Dark-field $(A, C$, and $E$ ) and bright-field $(B, D$, and $F)$ photomicrographs of three coronal sections $300 \mu \mathrm{m}$ apart through the lesion in case 564, an experimental monkey in which the SCN was destroyed completely on the right side but incompletely on the left. The finding of radioactive label (arrows) confirms the cell stain evidence, indicating that part of the left SCN had been spared. The scale in $F$ applies to $A$ to $F$. 

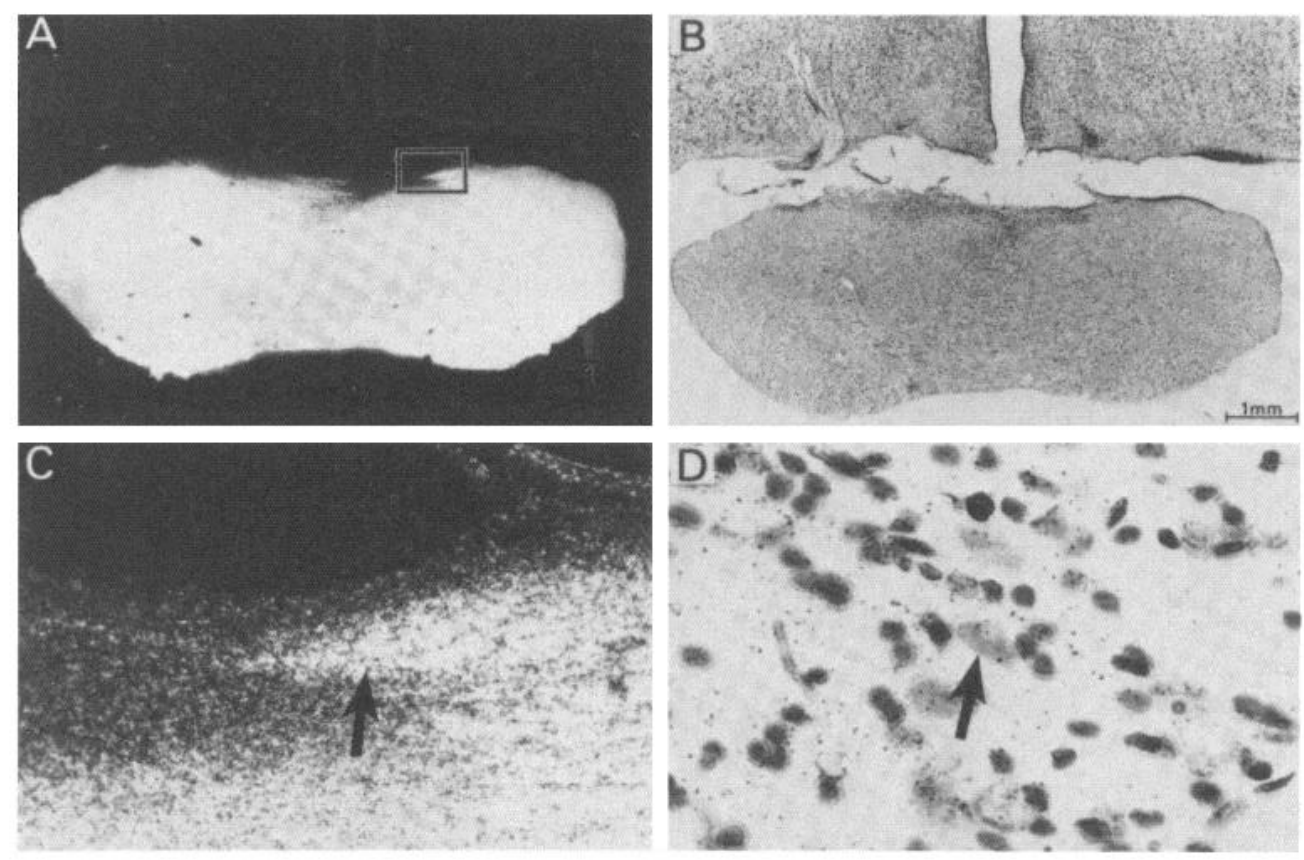

Figure 3. Dark-field $(A)$ and bright-field $(B)$ photomicrographs of a coronal section through the greatest extent of damage in case 585. Autoradiographic results in $A$ confirm the cell stain evidence in $B$ of complete destruction of the left SCN but reveal an intact remnant on the right (rectangle). The triangular cluster of darkly stained cells in $B$ within the right preoptic area 1 mm lateral to the third ventricle (see scale in $B$ ) represents displaced neurosecretory cells of the supraoptic nucleus. $C$, Dark-field photomicrograph of the area outlined in $A$ at a higher magnification $(\times 50)$ illustrating the spared portion $($ arrow) of the right SCN. $D$, High power $(\times 200)$, bright-field photomicrograph of the area indicated by the arrow in $C$. The arrow in $D$ demonstrates the termination of radioactive label (black dots) on neurons in this area.

bilaterally, and both supraoptic nuclei were partially damaged as well. Despite the presumed total destruction of the SCN, however, autoradiographic results revealed that there was an intact remnant of the RHP on the right (see Fig. 3, $A$ and $C$ ) which terminated on a small cluster of cells (see Fig. $3 D$ ). Although these cells were located within the chiasm at its dorsal edge, both their size and shape suggest that they were, in fact, spared neurons of the SCN. According to our estimates based on areal measurements, the sparing represents approximately $10 \%$ of the total RHP projection zone within the right SCN. However, the actual number of spared SCN neurons constitutes less than $10 \%$ of a normal SCN since the packing density of cells within the spared area was less than normal. No evidence for sparing was found within the left SCN.

\section{Melatonin physiology}

Control animals. Each of the two sham-operated animals (565 and 580) exhibited a large daily melatonin rhythm in diurnal lighting at 4 and 8 months after the operation (Fig. 4); the rhythm was characterized by high melatonin values at night. Although the day-to-day pattern for each animal was very consistent, there was considerable variation between animals both in the day and night concentrations of melatonin and in the amplitude of the rhythm. The quantitative differences in rhythms between the control animals are similar to those that we have observed previously among a large group of normal monkeys (Reppert et al., 1979).

The responses of the rhythm to constant light and to constant darkness in the controls were also the same as

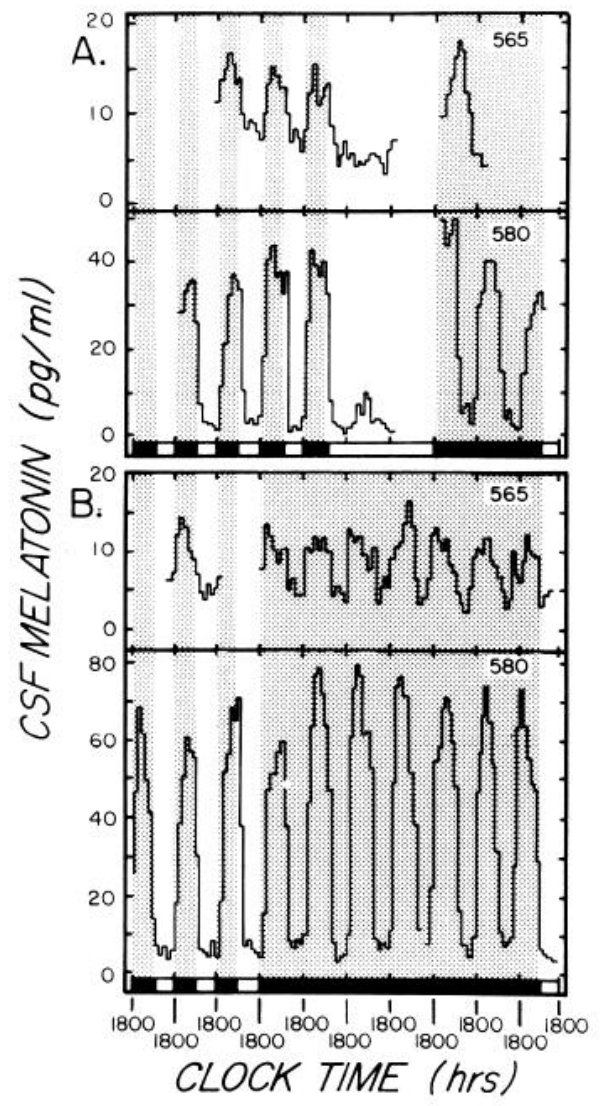

Figure 4. Patterns of CSF melatonin for the two shamoperated animals studied in various lighting schedules at 4 months $(A)$ and 8 months $(B)$ after the operation. 
that observed in normal animals (Perlow et al., 1979). That is, the daily rhythm was suppressed by constant light and persisted in constant darkness. It is worth noting that, in animal 580, melatonin concentrations showed a small increase at night when the lights were left on (Fig. $4 A$ ). Nonetheless, melatonin values at that time were clearly suppressed by light, with levels remaining at less than $25 \%$ of the normally high concentrations of melatonin found during the preceding dark period of diurnal lighting.

As can be seen in Figure $4 B$, the daily melatonin rhythm persisted in the control animals throughout the $6 \frac{1}{1 / 2}$-d period of study in constant darkness. The left column of Figure 5 illustrates the same melatonin data as in Figure $4 B$ converted to show the relative amplitude and timing (phase angle) of the daily rhythm. This figure demonstrates that, despite the quantitative differences between these two sham-operated monkeys noted in Figure $4 B$, the rhythms were quite similar qualitatively. Thus, the relative amplitudes of both increased slightly in constant darkness compared to diurnal lighting, and the relative timing of the two daily rhythms was practically the same as judged by the phase angle plot.
Animals with complete SCN ablation. Each of the animals with histologically confirmed complete bilateral ablation of the SCN exhibited a pattern of CSF melatonin which was strikingly different from that found in the control animals (Fig. 6). At the 4-month sampling period, visual inspection of the melatonin patterns revealed that neither of the animals with complete ablation exhibited a clear daily pattern of CSF melatonin concentrations in diurnal lighting nor were their melatonin concentrations at constant low levels. Instead, melatonin levels varied over a considerable range, from 7 to $20 \mathrm{pg} / \mathrm{ml}$ for animal 586 and from 4 to $15 \mathrm{pg} / \mathrm{ml}$ for animal 583. Furthermore, CSF melatonin levels were not suppressed consistently in either animal by constant light. In fact, the CSF melatonin concentrations for each were as high during the $2^{1 / 2}-\mathrm{d}$ period of constant light as they were at any time during the preceding 4-d period of study in diurnal lighting. In constant darkness, at the 4-month sampling period, a 24-hr rhythm to the melatonin pattern was not readily apparent for either animal by visual inspection of the data. Because of the short period of study in constant darkness during the first sampling period, time series analysis of the melatonin patterns was not attempted.
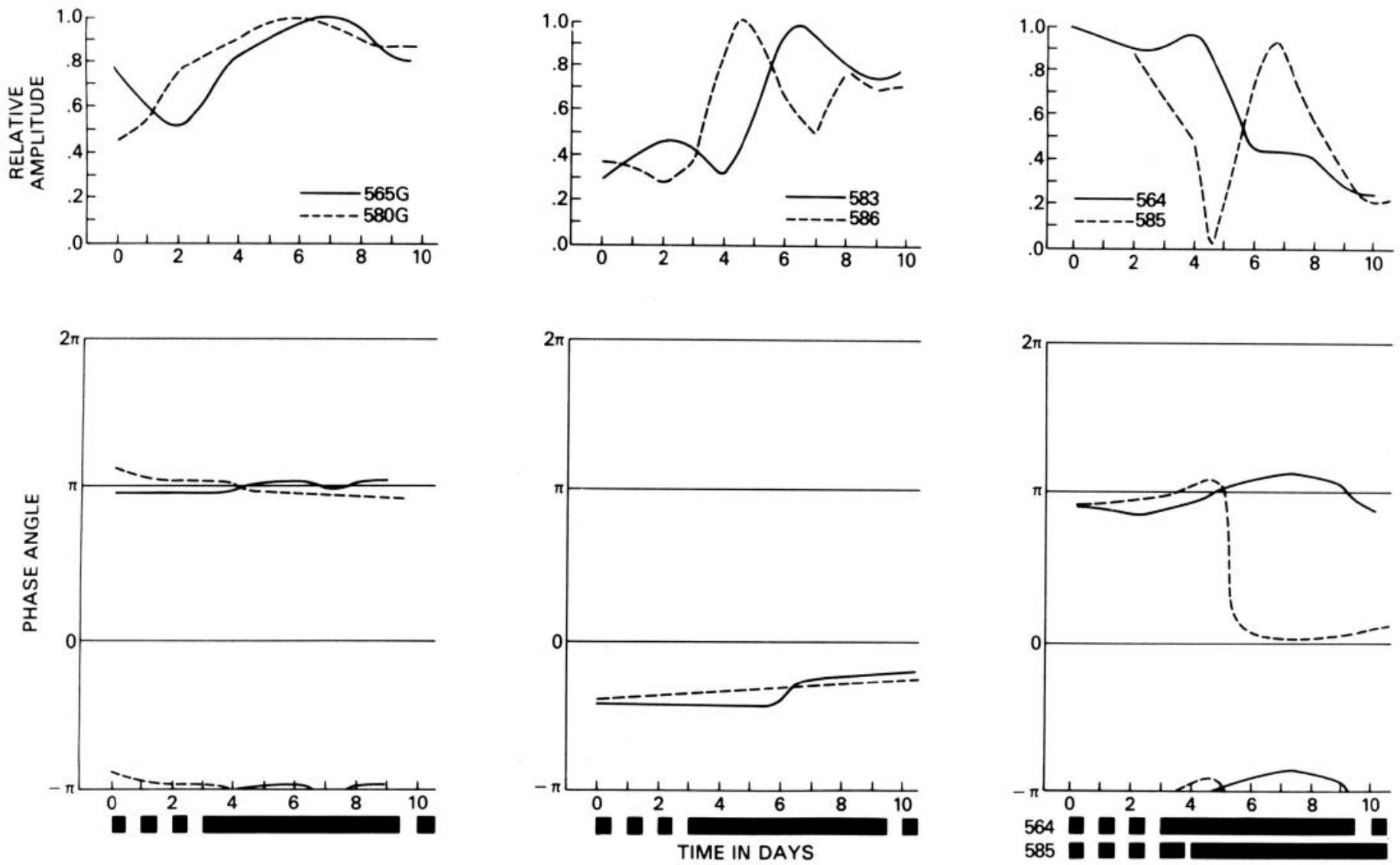

Figure 5. Relative amplitude and phase angle of the daily melatonin rhythm at the 8-month sampling period. The monkeys are grouped in pairs as follows: sham-operated (565 and 580) in the left column; animals with complete lesions (583 and 586) in the middle column; animals with incomplete SCN lesions (564 and 585) in the right column. The top row of the figure shows the relative amplitude plot of the circadian rhythm as a proportion of the maximum amplitude attained for each monkey. The bottom row shows the relative phase angle of the circadian rhythm, where 0 radian degrees corresponds to a peak amplitude of the cycle at noon and $\pm \pi$ radian degrees corresponds to a peak amplitude of the cycle at midnight. Hence, the circadian rhythm for the monkeys with complete lesions lagged approximately $0.7 \pi$ radian degrees (or $8.4 \mathrm{hr}$ ) behind the peak for the sham-operated monkeys. The phase angle plot is extended out to $+2 \pi$ so that the phase angle can be visualized without apparent discontinuities: the plot should actually occur on a cylinder so that $+\pi$ and $-\pi$ are identical. 


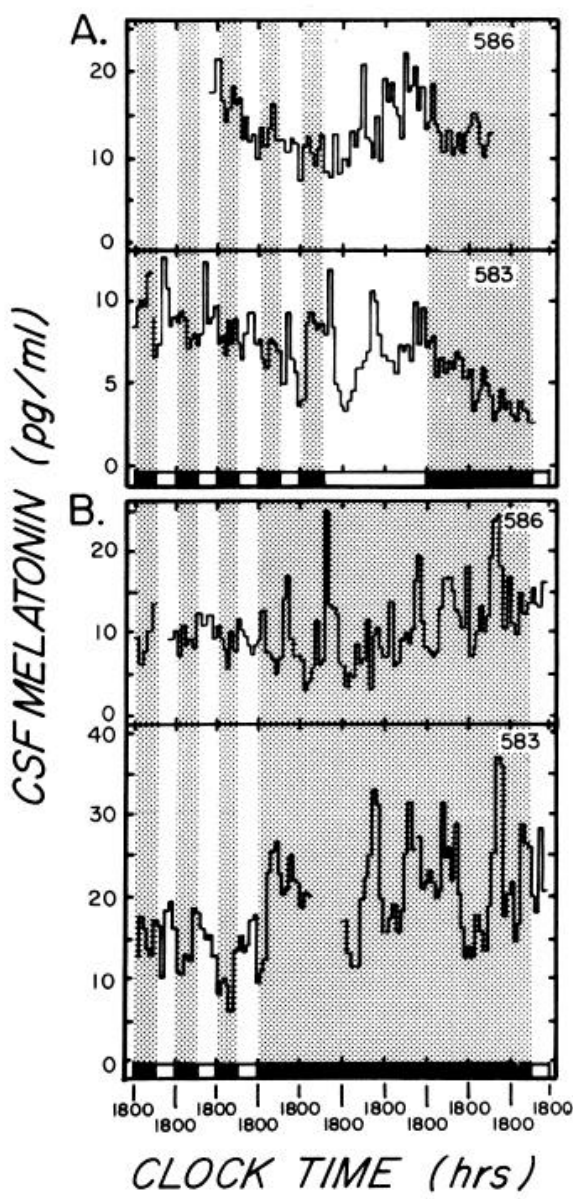

Figure 6. Patterns of CSF melatonin for the two animals with complete SCN ablation studied in various lighting schedules at 4 months $(A)$ and 8 months $(B)$ after the operation.

At 8 months after the operation, melatonin concentrations for each of the animals with complete ablation continued to show considerable variation. Interestingly, when each was placed in constant darkness, a clear increase in melatonin excursions occurred. Although a daily melatonin rhythm in constant darkness was not readily apparent by visual inspection of the data, spectral analysis revealed a $24-\mathrm{hr}$ component to the melatonin pattern for each animal in both diurnal lighting and constant darkness (Fig. 7).

Figure 5 shows the relative amplitude and phase angle for the animals with complete SCN damage. The previously mentioned increase in relative amplitude of the melatonin levels on placement into darkness is readily apparent. The relative timing of the cycle did not differ between these two monkeys, but as shown by the phase angle plot, their peak amplitude lagged $126^{\circ}(8.4 \mathrm{hr})$ behind that of the sham-operated animals.

Animals with partial SCN ablation. Partial SCN ablation (animals 564 and 585) had an intermediate effect on the melatonin rhythm (Fig. 8). Each of the animals with partial unilateral sparing exhibited a daily rhythm in CSF melatonin concentrations when studied in diurnal lighting at 4 and 8 months after the operation. The daily rhythm was similar in these animals to that found in control animals, with high melatonin concentrations gen- erally occurring during the dark period of the lighting cycle. However, the daily rhythm for the animals with partial SCN ablation was not as rigidly coordinated to the daily light-dark cycle as it was in the sham-operated control and normal animals. This was evidenced by high melatonin levels extending for up to $4 \mathrm{hr}$ into the light portion of the day, a pattern rarely observed in the normal animal.

As in the animals with complete SCN ablation, CSF melatonin levels of the animals with partial damage were not suppressed consistently by constant light (Fig. 8). The mean melatonin concentrations for animal 564 were the same during the period of constant light as they were during a comparable period of time in constant darkness. Similarly, the CSF melatonin levels for animal 585 were as high during the $2 \frac{1}{2}-\mathrm{d}$ period of constant light as they were during any of the preceding dark periods of diurnal lighting.

In animal 564, a daily rhythm in CSF melatonin concentrations persisted for the $6 \frac{1}{2} \mathrm{~d}$ of study in darkness. Although the phase relationship of the rhythm to time of day was similar to that manifested by the control animal,

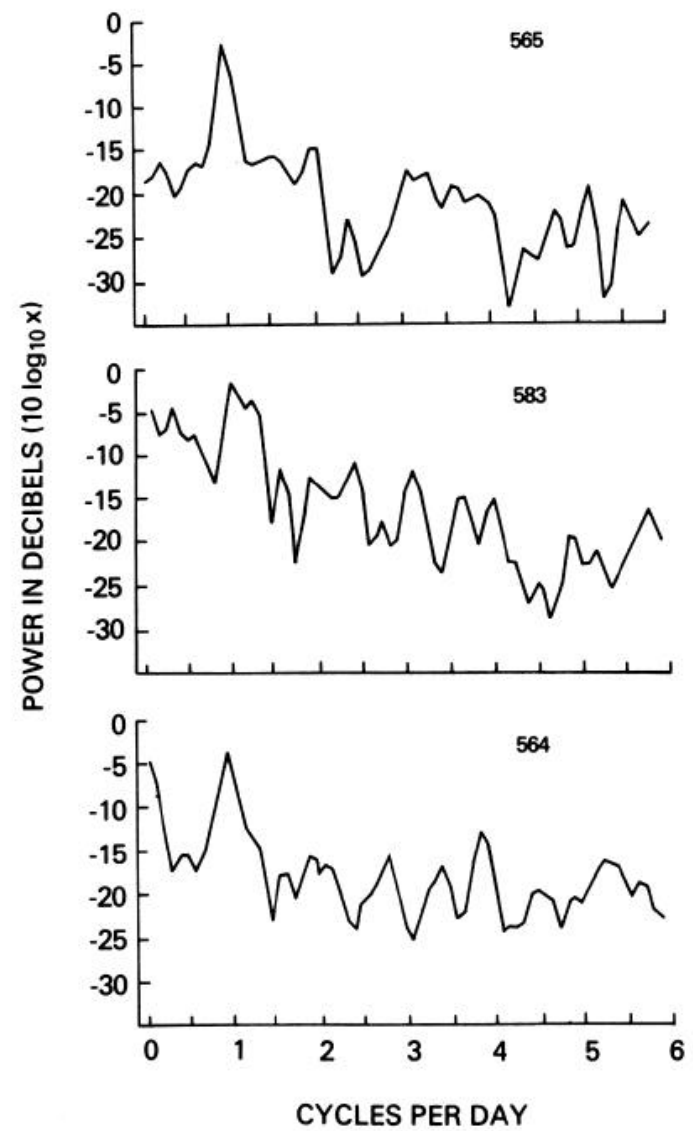

Figure 7. Power spectra for the melatonin measurements observed at the 8-month sampling period. The top panel shows the results for a sham-operated monkey (565), the middle panel, those for a monkey (583) with complete SCN ablation, and the bottom panel, those for a monkey (564) with partial SCN damage. Spectra are plotted in log (decibel) scale so that the variability of the estimate is virtually constant (i.e., the variability does not depend on the actual level of power in each frequency band). Peaks in the spectra at 1 cycle/d correspond to the frequency of a circadian rhythm. 


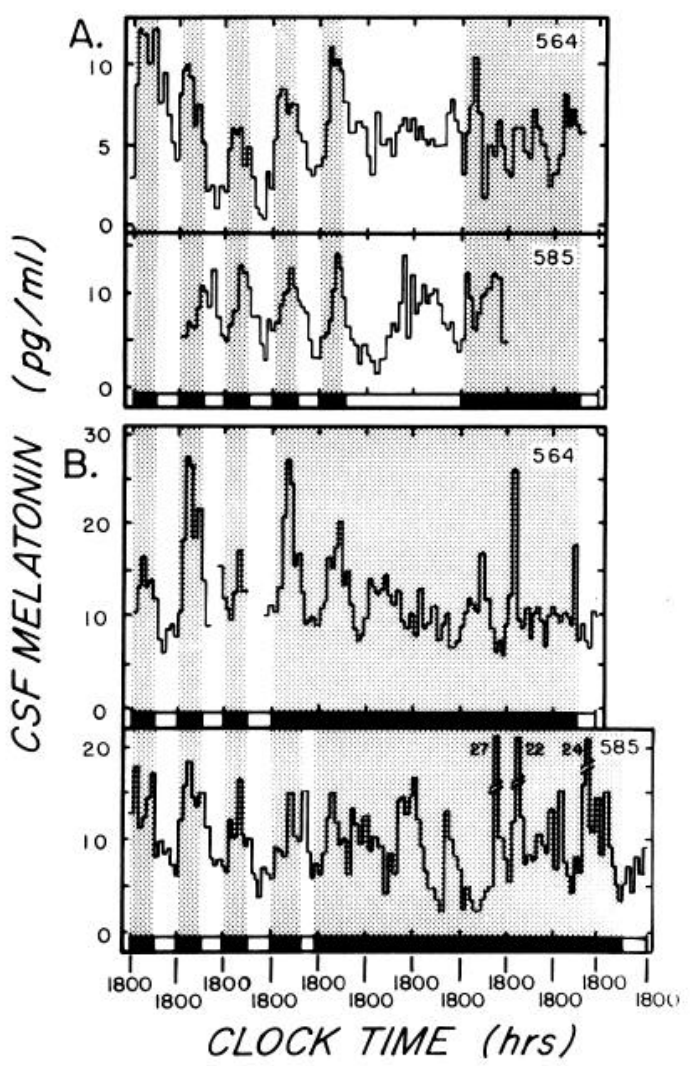

Figure 8. Patterns of CSF melatonin for the two animals with partial SCN ablation studied in various lighting schedules at 4 months $(A)$ and 8 months $(B)$ after the operation. When animal 585 was placed in darkness at the 8-month sampling period, the lights accidentally went on from 1100 to 1600 . The animal was kept in darkness for the subsequent $6 \frac{1}{2} \mathrm{~d}$.

there was a progressive dampening of the amplitude of the daily rhythm in this animal (Fig. 5).

When animal 585 was placed in darkness at the 8month sampling period, the lights accidentally went on from 1100 to $1600 \mathrm{hr}$. Thereafter, the animal was placed in constant darkness for the subsequent $6 \frac{1}{2} \mathrm{~d}$. Within 24 $\mathrm{hr}$ in darkness after the accidental period of light, the phase of the daily melatonin rhythm shifted acutely by about $10 \mathrm{hr}$ and this altered phase was maintained for the remaining period of study in darkness (Fig. 5).

\section{Cortisol physiology}

The CSF pattern of cortisol concentrations was remarkably similar in the control and experimental animals at 4 months (Fig. 9) and at 8 months (Fig. 10) after the operation. The pattern was characterized by a clear daily rhythm in diurnal lighting, with high cortisol levels occurring prior to lights-on in the morning. Although there were some differences in the amplitude of the daily rhythm among the animals and for individual animals between the 4- and 8-month sampling periods, the rhythm persisted in all animals in constant light and for the two sampling periods in constant darkness. The timing of the rhythm also was the same among all of the animals and was unaltered by either constant light or constant darkness (Fig. 11).

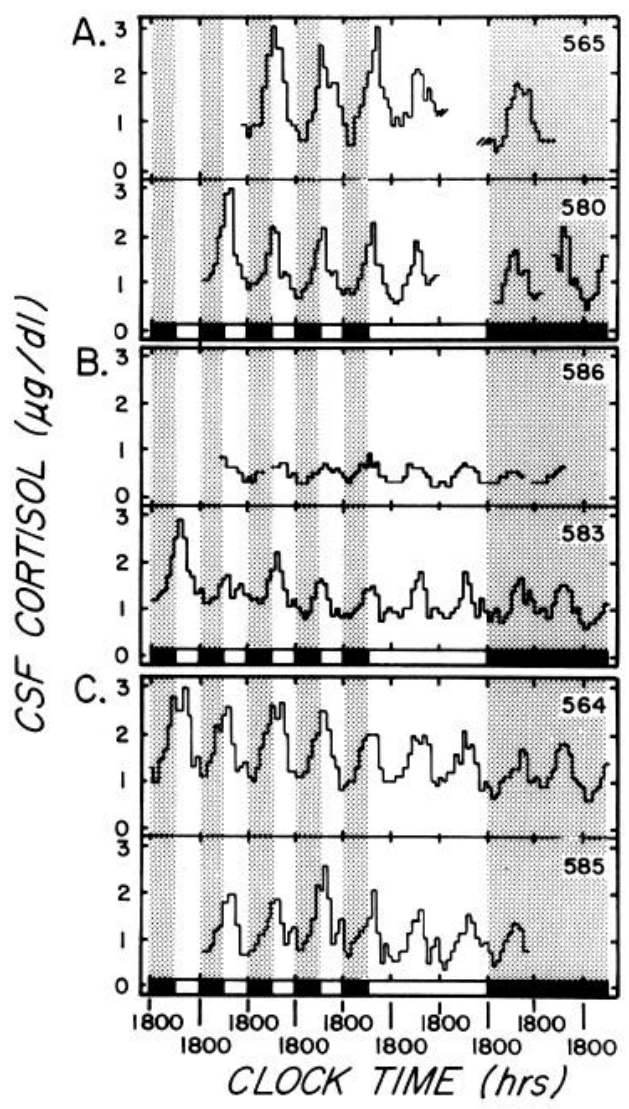

Figure 9. Patterns of CSF cortisol for the sham-operated animals $(A)$ and the animals with complete $(B)$ and partial $(C)$ $\mathrm{SCN}$ damage at 4 months after the operation.

\section{Discussion}

The results of this study reveal striking differences between the effects of SCN ablation on the daily melatonin and cortisol rhythms in the monkey. On the one hand, SCN damage resulted in marked alterations in the melatonin rhythm, in its coordination by environmental lighting, in the acute suppressive effect of light on melatonin secretion, and in its endogenous nature. On the other hand, little alteration was observed in the daily cortisol rhythm. These findings will be discussed in detail in the following sections.

Melatonin physiology. Based on the results of studies of pineal $N$-acetyltransferase (NAT), the enzyme that controls melatonin production in the rat, and CSF melatonin in the rhesus monkey, there are two effects of light which normally act to control the expression of the daily melatonin rhythm (Reppert and Klein, 1980); in the rodent, both effects are transmitted from the eye to SCN via the RHP (Klein and Moore, 1979). The first effect is entrainment of the endogenous melatonin rhythm to the 24-hr day. The response of the rhythm to the entraining effect of lighting is slow as evidenced by an earlier finding in the monkey that it takes a few days for the melatonin rhythm to resynchronize to $12-\mathrm{hr}$ phase shifts in the daily light-dark cycle (Reppert et al., 1981). The second effect of light is acute suppression of melatonin production. Thus, exposure to light at night when CSF melatonin levels are normally high results in a rapid decrease in 


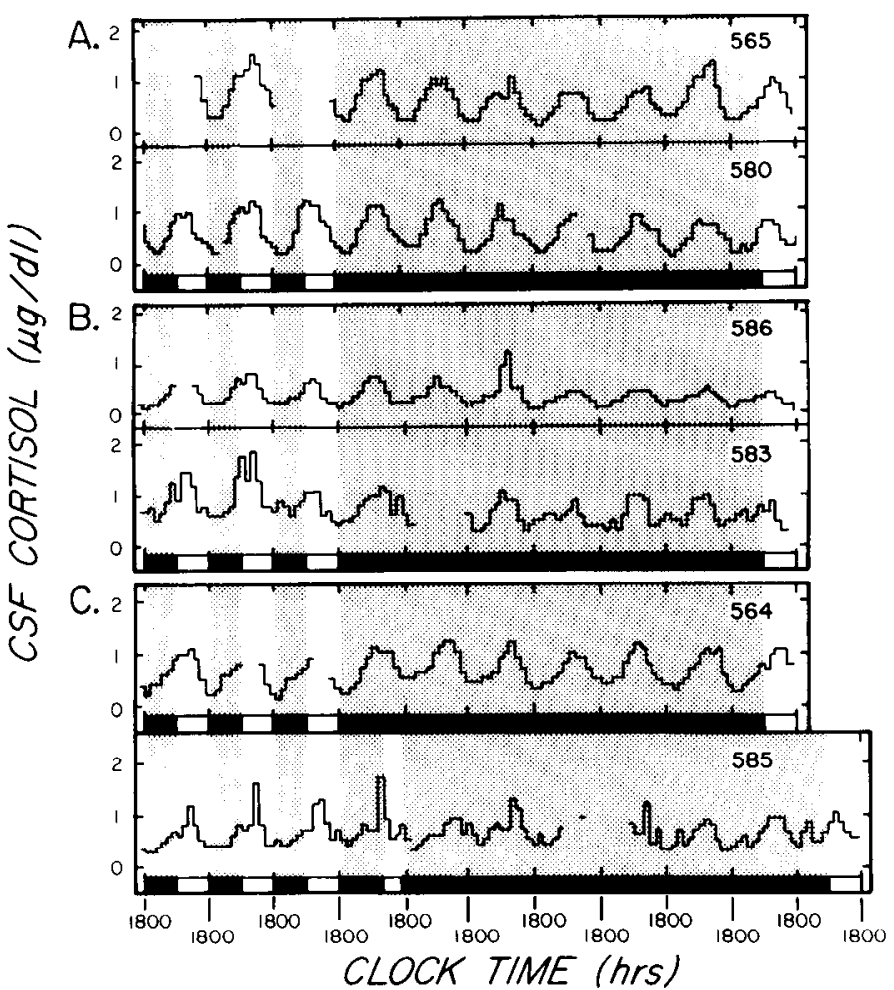

Figure 10. Patterns of CSF cortisol for the sham-operated animals $(A)$ and the animals with complete $(B)$ and partial $(C)$ SCN damage at 8 months after the operation. When animal 585 (C) was placed in darkness at the 8-month sampling period, the lights accidentally went on from 1100 to $1600 \mathrm{hr}$. Thereafter, the animal was kept in darkness for $6 \frac{1 / 2}{d}$.

melatonin levels to low daytime values (Reppert et al., 1981). In addition, when monkeys are maintained in a lighted environment, the normally high night-time levels of melatonin are suppressed for the duration of light exposure. The combination of these two effects of light determines that high melatonin levels occur only at night in darkness in a normal light-dark cycle.

In the monkeys with complete SCN ablation, the normal relationship of the melatonin rhythm to the daily lighting schedule was disrupted markedly. At both the 4and 8-month sampling periods, no clear 24-hr component to the CSF melatonin patterns was evident in diurnal lighting for either animal with complete SCN damage by visual inspection of the data. However, spectral analysis of the melatonin patterns at the 8-month period did reveal a daily rhythm in these animals. It was characterized by high values during the day, in contrast to the normal situation where high levels occur at night.

In the animals with partial SCN ablation, on the other hand, the coordinating effect of the daily light-dark cycle appeared for the most part to be preserved. This is quite remarkable in view of the evidence that, in animal 585, less than $5 \%$ of all SCN neurons were spared. Thus, it would appear that the coordinating effect of environmental lighting on the daily melatonin rhythm in the monkey can be sustained by only a very small portion of the SCN-RHP complex.

In contrast to differences between animals with partial and complete SCN damage in the coordination of the melatonin rhythm by lighting, the suppressive effect of light on melatonin production was blocked uniformly in all cases. This finding suggests either that the light suppression effect requires more SCN tissue than does rhythm coordination or that the light suppression effect involves a second anatomical site, one that was equally damaged in all four experimental monkeys. Because of the limited number of animals studied with partial lesions, we could not distinguish between these two possibilities.

A primary aim of this study was to examine the effect of SCN ablation on the endogenous expression of the melatonin rhythm. In the rat, the pineal NAT rhythm, as monitored under conditions of both diurnal lighting and constant darkness, is abolished consistently following SCN ablation (Moore and Klein, 1974; Klein and Moore, 1979); NAT values remain at constant low levels throughout a 24-hr period. The effects of complete SCN ablation on the melatonin rhythm in the monkey were quite different. First, the melatonin levels fluctuated considerably throughout the 24-hr day in the animals with complete ablation. Second, and more surprising, a 24-hr component in constant darkness appeared in both of these animals 8 months after surgery.

These species differences may be artifactual, however. In the rodent studies, data were derived from a population of animals, and relatively few time points were monitored throughout the day. In the monkey studies, by contrast, melatonin was sampled continuously in the same subject twice for extended periods after surgery. Studies involving more frequent monitoring of the melatonin rhythm in the rat, a difficult task in the small rodent, are required before it can be concluded that these are true species differences in the response of the melatonin rhythm to SCN ablation.

There are at least three possible explanations for the persistence of an apparent 24-hr melatonin rhythm in the monkeys with complete SCN ablation. First, a small number of spared SCN neurons within the SCN limits may have escaped identification. This seems unlikely because our histological conclusions were based not only on bright-field examination of the lesions but also on autoradiographic tracings of the RHP within the SCN. The value of this combined histological approach is evident from the data on animal 585. Based on the histological examination of the brain under bright-field illumination, we had concluded tentatively that both SCN were destroyed completely. The autoradiographic results, however, demonstrated a small number of surviving RHP fibers, which led to the discovery of a few spared SCN neurons.

A second possibility is that the daily care and food delivery system used in the studies contributed to the apparent 24-hr rhythm in melatonin values manifested in constant darkness. Parenthetically, it is important to emphasize that the food delivery system did not constitute a rigidly controlled, restricted period of feeding. Rather, the animals could eat whenever they chose; what was constant was the time of day that fresh food was delivered, which was coincident with the daily care and cleaning.

In the intact monkey, we have shown that this care and food delivery system does not affect either the expression or the phase of the daily melatonin rhythm 

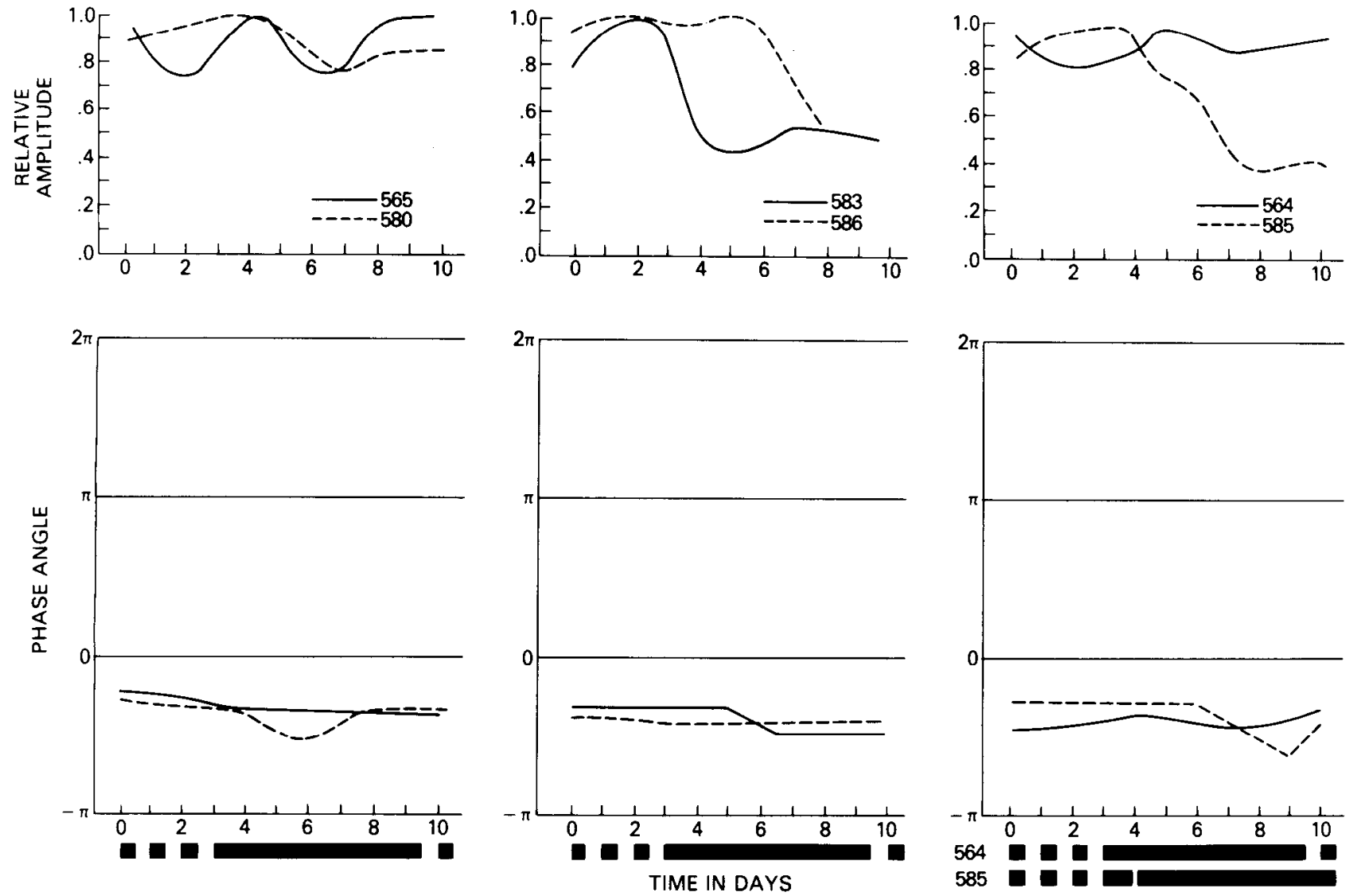

Figure 11. Relative amplitude and phase angle of the daily cortisol circadian rhythm at the 8-month sampling period. The relative amplitude is shown in the top row of the figure and the corresponding phase angle plots are in the bottom row (see the legend to Fig. 5). The phase angle diagram shows that the cortisol rhythm peaks regularly at about $0800 \mathrm{hr}$.

measured for several days in constant darkness (Reppert et al., 1981). It is possible, however, that the potential cue could have artificially generated a daily melatonin rhythm in the animals with complete SCN ablation. In support of this possibility is the finding that the rhythmic increase in melatonin levels occurred together in both animals.

A third explanation is that the rhythm represents the expression of an endogenous oscillator located outside of the SCN region. If so, our finding could represent either an unmasking of a circadian oscillator that normally is coupled to or under control of the SCN or the take over of control of pineal activity by a normally independent oscillator.

It is important to note that the normal endogenous expression of the CSF melatonin rhythm appeared to be at least partially preserved following partial SCN ablation. This is exemplified by case 564 where about $35 \%$ of the SCN-RHP complex was spared unilaterally. In this animal, the daily melatonin rhythm persisted for $6^{1 / 2} \mathrm{~d}$ of study in darkness and the phase of the rhythm was similar to that manifested by the control animals. However, there was a clear, progressive dampening of the amplitude of the rhythm (Fig. 5).

The pattern of melatonin production exhibited in the other animal (585) with partial ablation is enigmatic. Although a daily rhythm persisted in constant darkness, there was an acute change in the phase of the rhythm which occurred shortly after the accidental period of light exposure (Fig. 5). This phase alteration may have been due to a direct phase setting of the rhythm in response to the accidental period of light. This interesting possibility is difficult to assess, however, since we do not have sufficient information concerning the response of the melatonin rhythm in the intact monkey to varying phase alterations in environmental lighting.

Cortisol physiology. In contrast to the marked effects of SCN ablation on the CSF melatonin rhythm, there was no appreciable effect of either partial or complete SCN damage on the daily pattern of CSF cortisol in the monkey. Furthermore, neither the phase relationship of the rhythm to the light-dark cycle nor the persistence of the daily rhythm in constant lighting conditions (i.e., constant light and constant darkness) was altered by the lesions. These data suggest that, in the rhesus monkey, a circadian oscillator located outside and independent of the SCN is involved in the generation of the cortisol rhythm and in its coordination by lighting.

It should be noted that the potential effect of the daily care and food delivery system in our studies qualifies somewhat the assumption, based on the persistence of the rhythm in constant lighting conditions, that the endogenous expression of the cortisol rhythm was unaffected by SCN ablation. Even though we have shown that this time cue does not affect the endogenous expression of the cortisol rhythm in the intact monkey, perhaps it acted to generate or coordinate the daily cortisol rhythm following SCN ablation. Against the possibility 
that the cue generated the rhythm is the finding that, in all cases and under all three lighting conditions (diurnal lighting, constant light, and constant darkness), the rhythmic increase in cortisol occurred 4 to $6 \mathrm{hr}$ prior to the time of daily care and food delivery.

The notion that more than one circadian pacemaker is involved in the circadian time-keeping system of primates is supported by the results of studies in man. When human subjects are placed in an environment free of time cues, circadian rhythms in sleep-wake cycles and core body temperature manifest different period lengths, suggesting that each is generated by a separate circadian pacemaker (Aschoff, 1980). Interestingly, the circadian cortisol rhythm under the above conditions has a fixed phase relationship to the body temperature rhythm, suggesting that the cortisol and temperature rhythms in primates are generated by the same circadian pacemaker.

The hypothesis that at least two separate circadian pacemakers are involved in the circadian time-keeping hierarchy of primates has been examined recently in the squirrel monkey by Moore-Ede et al. (1980). These investigators found that, whereas SCN lesions disrupt circadian rhythms in rest-activity and in eating and drinking, a clear body temperature rhythm, confirmed by spectral analysis, persists. In addition, despite the complete SCN ablation, the body temperature rhythm could be entrained precisely to the light-dark cycle. Hence, our findings of spared cortisol rhythm after SCN ablation in the rhesus monkey and of unaltered phase relationships to the light-dark cycle (see also Spies et al., 1979) may be related to the finding of spared body temperature rhythm following SCN lesions in the squirrel monkey. Study of the effects of SCN lesions on the two rhythms in the same and in several different primate species is needed to determine whether the two are indeed generated by the same circadian oscillator outside of the SCN region and whether the relationship holds true for primates in general.

In the rodent, the entraining effect of light on circadian rhythms is transmitted from the eye to the SCN primarily via the RHP (Moore, 1979). Since this pathway is destroyed in monkeys with complete SCN ablation, any entraining effect of light on the endogenous oscillator which controls the cortisol and temperature rhythms in the primate probably involves light transmission to the brain by other retinal pathways. Recently a second retinohypothalamic projection in the rat has been described in which dendrites of the lateral hypothalamus extend into the optic tract and form synapses with axons arising in the retina (Riley et al., 1980). It is possible that this second pathway exists in the primate as well and is involved in the regulation of both the cortisol and body temperature rhythms.

Interestingly, the minimal effect of SCN ablation on the cortisol rhythm in the monkey contrasts with the marked effects of ablation on the corticosterone rhythm in the rat. Under conditions of diurnal lighting, the daily corticosterone rhythm in this species has been disrupted consistently following electrolytic lesions of the SCN (Moore and Eichler, 1972; Krieger et al., 1977; Raisman and Brown-Grant, 1977). No data are yet available, however, concerning the effects of SCN damage on the endogenous expression of the rodent corticosterone rhythm.
In summary, the results of our studies support the notion that the circadian time-keeping system in the primate involves complex interactions among several circadian oscillators. The SCN in the rhesus monkey clearly is important for the generation, photic entrainment, and photic suppression of the melatonin rhythm. However, oscillators located outside of the SCN area may control the circadian expression of the cortisol rhythm and perhaps even the melatonin rhythm in the absence of the SCN. Further studies are needed in order to establish the precise role of the SCN in the circadian time-keeping hierarchy of primates and to determine whether our findings in the rhesus monkey are applicable to the physiology of human circadian rhythms.

\section{References}

Aschoff, J. (1980) The circadian system in man. In Neuroendocrinology, D. T. Krieger and J. C. Hughes, eds., pp. 77-83, Sinauer Associates, Sunderland, MA.

Bingham, C., M. D. Godfrey, and J. W. Tukey (1967) Modern technique of power spectrum estimation. IEEE Trans. Audio Electroacoust. $A U-15$ : 56-66.

Bloomfield, P. (1976) Fourier Analysis of Time Series: An Introduction, John Wiley and Sons, New York.

Cowan, W. M., D. I. Gottlieb, A. E. Hendrickson, J. L. Price, and T. W. Woolsey (1972) The autoradiographic demonstration of axonal connections in the central nervous system. Brain Res. 37: 21-51.

Gentleman, W. M., and G. Sande (1966) Fast Fourier transforms-for fun and profit. In 1966 Fall Joint Computer Conference, AFIPS Conference Proceedings, Vol. 29, pp. 563-578.

Klein, D. C., and R. Y. Moore (1979) Pineal $N$-acetyltransferase and hydroxyindole- $O$-methyl-transferase: Control by the retina hypothalamic tract and the suprachiasmatic nucleus. Brain Res. 174: 245-262.

Krieger, D. T., H. Hauser, and L. C. Krey (1977) Suprachiasmatic nuclear lesions do not abolish food-shifted circadian adrenal and temperature rhythmicity. Science 197: 398-399.

Moore, R. Y. (1973) Retinohypothalamic projection in mammals: A comparative study. Brain Res. 49: 403-409.

Moore, R. Y. (1979) The anatomy of central neural mechanisms regulating endocrine rhythms. In Endocrine Rhythms, D. T. Krieger, ed., pp. 63-87, Raven Press, New York.

Moore, R. Y., and V. B. Eichler (1972) Loss of circadian adrenal corticosterone rhythm following suprachiasmatic lesions in the rat. Brain Res. 42: 201-206.

Moore, R. Y., and D. C. Klein (1974) Visual pathways and the central neural control of a circadian rhythm in pineal serotonin $N$-acetyltransferase activity. Brain Res. 71: 17-33.

Moore-Ede, M. C., R. Lydic, C. A. Czeizler, C. A. Fuller, and H. E. Albers (1980) Structure and function of suprachiasmatic nuclei (SCN) in man and non-human primates. Soc. Neurosci. Abstr. 6: 708.

Orr, W. C., and H. J. Hoffman (1974) A 90-min. cardiac biorhythm: Methodology and data analysis using modified periodograms and complex demodulation. IEEE Trans. Biomed. Eng. $B M E-21$ : 130-143.

Perlow, M. J., S. J. Enna, P. O'Brian, H. J. Hoffman, and R. J. Wyatt (1979) Cerebrospinal fluid gamma-aminobutyric acid: Daily rhythm and response to haloperidol. J. Neurochem. 32: 265-268.

Perlow, M. J., S. M. Reppert, L. Tamarkin, R. J. Wyatt, and D. C. Klein (1980) Photic regulation of the melatonin rhythm: Monkey and man are not the same. Brain Res. 182: 211-216. Perlow, M. J., S. M. Reppert, R. M. Boyar, and D. C. Klein (1981) Daily rhythms in cortisol and melatonin in primate CSF. Neuroendocrinology 32: 193-196. 
Raisman, G., and K. Brown-Grant (1977) The "suprachiasmatic syndrome": Endocrine and behavioral abnormalities following lesions of the suprachiasmatic nuclei in the female rat. Proc. R. Soc. Lond. (Biol.) 198: 297-314.

Reppert, S. M., and D. C. Klein (1980) Mammalian pineal gland: Basic and clinical aspects. In The Endocrine Functions of the Brain, M. Motta, ed., pp. 327-371, Raven Press, New York.

Reppert, S. M., M. J. Perlow, L. Tamarkin, and D. C. Klein (1979) A diurnal rhythm in primate cerebrospinal fluid. Endocrinology 104: 295-301.

Reppert, S. M., M. J. Perlow, and D. C. Klein (1980) Cerebrospinal fluid melatonin. In Neurobiology of Cerebrospinal Fluid, J. H. Wood, ed., pp. 579-589, Plenum Press, New York.

Reppert, S. M., M. J. Perlow, L. Tamarkin, D. Orloff, and D. C.
Klein (1981) The effects of environmental lighting on the daily melatonin rhythm in primate cerebrospinal fluid. Brain Res. 223: 313-323.

Riley, J. N., J. P. Card, and R. Y. Moore (1980) A retinal projection to the lateral hypothalamus in the rat. Soc. Neurosci. Abstr. 6: 266.

Rollag, M. D., and G. D. Niswender (1976) Radioimmunoassay of serum concentrations of melatonin in sheep exposed to different lighting regimens. Endocrinology 98: 482-488.

Rusak, B., and I. Zucker (1979) Neural regulation of circadian rhythms. Physiol. Rev. 59: 449-526.

Spies, H. G., R. L. Norman, and A. E. Buhl (1979) Twenty-fourhour patterns in serum prolactin and cortisol after partial and complete isolation of the hypothalamic-pituitary unit in rhesus monkeys. Endocrinology 105: 1361-1368. 